\title{
BELA TAGARELA: APLICAÇÃO MÓVEL PARA COMUNICAÇÃo AUMENTATIVA E ALTERNATIVA
}

\author{
Bela Tagarela: Mobile Application for Augmentative and Alternative \\ Communication
}

\author{
Fernanda de Paula Silva* \\ Suammy Priscila Rodrigues Leite Cordeiro** \\ Selma Terezinha Milagre ${ }^{* * *}$
}

\begin{abstract}
Resumo: Este trabalho apresenta o desenvolvimento de um aplicativo para dispositivos móveis de Comunicação Aumentativa e Alternativa (CAA) voltado para crianças e adolescentes com impossibilidades de comunicação verbal. O objetivo deste artigo é discorrer sobre o processo de criação do aplicativo, suas funcionalidades e seu diferencial. Há vários recursos de CAA disponíveis, porém muitos deles são insuficientes para seus usuários em certos aspectos, seja pela quantidade reduzida de expressões ou pela falta de portabilidade do recurso. Com o intuito de minimizar esta problemática, inicialmente foi feita uma pesquisa de fundamentação teórica acerca da área de Tecnologia Assistiva (TA), bem como uma revisão de ferramentas de CAA para celulares e tablets já existentes. Após tal pesquisa, foi desenvolvida uma aplicação para comunicação por dispositivos móveis. Obteve-se, como resultado deste trabalho a criação de Bela Tagarela, aplicativo de CAA focado na facilidade de uso e atratividade para o usuário. Com este trabalho, espera-se que haja um constante avanço no desenvolvimento de Tecnologia Assistiva de baixo custo e acessíveis à população com o intuito de reduzir as limitações de pessoas com deficiência.
\end{abstract}

Palavras-chave: Tecnologia Assistiva. Comunicação Aumentativa. Comunicação Alternativa. Inclusão. Aplicação móvel.

Abstract: This paper presents the development of an Augmentative and Alternative Communication (AAC) application for mobile devices, designed for children and teenagers with verbal communication disabilities. The goal of this paper is to expatiate about the creation

\footnotetext{
* Doutoranda e Mestre em Engenharia Biomédica (UFU), estudante da Pós-graduação em Educação Profissional e Tecnológica Inclusiva (IFTM), Pós-graduada em Atendimento Educacional Especializado e Psicomotricidade (FAVENI), Graduada em Pedagogia (FACIBRA) e em Sistemas para Internet (IFTM), Docente na Rede Municipal de Ensino de Uberlândia/MG. ORCID: 0000-0002-3988-0485. Lattes: http://lattes.cnpq.br/8541971662060122. E-mail: ps.fernanda29@gmail.com.

** Doutoranda em Educação pela Universidade de Lisboa, Mestre em Linguística pela Universidade Federal do Mato Grosso, Graduada em Pedagogia pela Universidade Estadual de Pernambuco, Docente no Instituto Federal do Mato Grosso (IFMT). ORCID: 0000-0002-3019-5799. Lattes: http://lattes.cnpq.br/9114383823113511. Email: suammy.cordeiro@ifmt.edu.br.

*** Doutora em Engenharia Elétrica (USP/São Carlos), Mestre em Engenharia Elétrica (UEL), Pós-graduada em Análise de Sistemas (UFU), Graduada em Engenharia Química (UFU). Docente (UFU) e Coordenadora do Núcleo de Inovação e Avaliação Tecnológica em Saúde (NIATS). ORCID: 0000-0002-0807-9839. Lattes: http://lattes.cnpq.br/4980687835840176. E-mail: stmilagre@gmail.com
} 
process of application, its functionalities and differential. There are several features of CAA available, but a lot of them are insufficient for the users, either by the reduced quantity of the expressions or for lack of resource portability. With the intention to minimize this problematic, a research on the Assistive Technology theoretical foundation (AT) was initially made, as well as a tool revision of AAC for mobile and tablets that are already available. After this research, an application for communication by device mobile was developed. As a result of this research, Bela Tagarela was created, an AAC application focused on making the use easier and more attractive for the user. With this paper, it is expected that there will be a constant progress towards low cost and accessible Assistive Technology development, in order to reduce the limitation of people with disabilities.

Keywords: Assistive Technology. Augmentative Communication. Alternative Communication. Inclusion. Mobile application.

\section{Introdução}

Por meio da vivência como docente na rede pública de ensino, na qual a inclusão ocorre em grande parte de forma deficitária, gerando apenas a integração do aluno com deficiência na sala de aula regular, sem envolvê-lo verdadeiramente na dinâmica da aula em igualdade de expressão e comunicação com seus colegas de classe, surgiu o questionamento de como seria possível tornar meios de Comunicação Aumentativa e Alternativa (CAA) mais funcionais, portáteis e atrativos para seus usuários e para as pessoas do seu convívio.

Quando se fala de tecnologias inclusivas, é notável a necessidade de abranger o tema não somente à escola, mas a toda sociedade, com o intuito de ofertar cada vez mais autonomia a pessoas com impossibilidade de fala em todos os ambientes da sua rotina. Nota-se a crescente necessidade de desenvolver meios para a inclusão com o objetivo de gerar autonomia a essas pessoas, independentemente de suas limitações e particularidades.

Existem diversas ferramentas de Tecnologia Assistiva (TA) empregadas como alternativa de comunicação e interação, dentre as quais há uma área denominada Comunicação Aumentativa e Alternativa (CAA), utilizada para "dar voz" a essas pessoas para que expressem seus desejos, preferências, anseios e necessidades. Conforme descreve Lauand (2005), o objeto da TA é dispor de uma ampla variedade de recursos destinados a dar suporte (mecânico, elétrico, eletrônico, computadorizado, etc.) às pessoas com deficiência física, visual, auditiva, intelectual ou múltipla, podendo ser: cadeira de rodas, próteses, órteses, uma série infindável de adaptações, aparelhos e equipamentos nas mais diversas áreas de necessidade pessoal (comunicação, alimentação, transporte, educação, lazer, esporte, trabalho, elementos arquitetônicos, entre outras).

O objetivo desta pesquisa é apresentar uma aplicação desenvolvida para dispositivos móveis (celulares e tablets) capaz de ser utilizada como ferramenta de apoio para a CAA, tanto no ambiente escolar quanto no ambiente doméstico e social em geral, que se diferencie pela atratividade e simplicidade de utilização. A fim de alcançar o propósito desta pesquisa, foram estipulados os objetivos específicos: (1) especificar as necessidades de uma aplicação de dispositivos móveis de CAA; (2) apresentar a documentação referente aos diagramas de casos de uso, de atividades, além dos requisitos funcionais e não-funcionais e (3) apresentar as características da aplicação desenvolvida e seu comportamento de acordo com o uso.

Com o intuito de criar uma aplicação de CAA para uso em celulares e tablets, visando à ampliação de uma habilidade funcional deficitária, foi determinado que o desenvolvimento seria para dispositivos móveis e não para computadores, devido, por exemplo, à portabilidade 
e uso difundido de tais equipamentos na atualidade. Em consequência disso, espera-se que os resultados deste estudo possam contribuir com as discussões atuais e futuras em torno da temática de possibilidades de CAA acessíveis para a população.

\section{Comunicação Aumentativa e Alternativa}

A CAA é definida como a integração de símbolos (gestos, sinais, imagens), recursos (pranchas, álbuns, softwares), técnicas (apontar, acompanhar, segurar) e estratégias (uso de histórias, brincadeiras, imitações), visando auxiliar uma gama de pessoas com impossibilidades de comunicação (SANTAROSA et al., 2010), possibilitando sua interação e podendo, assim, expressar mais claramente seus sentimentos e desejos. Pode funcionar tanto como um substituto permanente à fala, quanto como um auxílio para determinadas dificuldades de comunicação. Vale ressaltar que a CAA auxilia não só a interação por meio da fala, mas também a interação por meio da escrita e da leitura, conforme apresentado pela Associação Americana de Fala, Linguagem e Audição, em inglês American Speech-Language-Hearing Association (AMERICAN SPEECH-LANGUAGE-HEARING ASSOCIATION, 2019). A capacidade de se comunicar por meio da linguagem é uma característica exclusivamente humana que nos diferencia dos outros primatas (ARGYLE, 1976).

A CAA trata-se de uma área da Tecnologia Assistiva (TA) que ajuda indivíduos impossibilitados, com prejuízo de fala, com prejuízos em sua comunicação ou capacidade de falar ou escrever que utilizam de escrita funcional. Este tipo de comunicação pode ser feito utilizando cartões e pranchas alfabéticas, vocalizador ou outros recursos para auxiliar nas habilidades de compreensão e expressão (FONTE, 2016). A CAA conta com diversos aspectos que devem ser analisados quando é adotada para a interação social, objetivando compensar a ausência de fala ou facilitar a comunicação, podendo ser utilizada como um instrumento de auxílio permanente ou provisório, para indivíduos com prejuízos ou incapacidades na fala (AMERICAN SPEECH-LANGUAGE-HEARING ASSOCIATION, 2019).

Conforme apresentado pela Associação Americana de Fala, Linguagem e Audição (AMERICAN SPEECH-LANGUAGE-HEARING ASSOCIATION, 2019), o público-alvo para a utilização de recursos de CAA é abrangente, seja para deficiências congênitas: Transtorno do Espectro Autista (TEA), Paralisia Cerebral (PC), Transtornos Globais do Desenvolvimento (TGD), deficiência intelectual, apraxia de fala e síndromes genéticas, entre outros. Seja para deficiências adquiridas: Acidente Vascular Cerebral (AVC), Traumatismo Crânio Encefálico (TCE), doenças neurodegenerativas (Esclerose Lateral Amiotrófica (ELA), deficiências após cirurgias (glossectomia, laringectomia) e condição temporária (intubação), entre outras.

\section{Bela Tagarela: o desenvolvimento de uma aplicação móvel para CAA}

A ferramenta utilizada para o desenvolvimento do aplicativo foi baseada na IBM Rational Unified Process (IRUP) ${ }^{1}$, que é um processo de engenharia de software que fornece uma abordagem disciplinada para assegurar a produção de qualquer programa de computador ou de dispositivos móveis dentro de seus requisitos (KRUCHTEN, 2004). Sendo assim, o desenvolvimento se deu seguindo as seguintes etapas.

\footnotetext{
${ }^{1}$ Mais informações no documento Rational Unified Process: <https://www.ibm.com/developerworks/rational/library/content/03July/1000/1251/1251_bestpractices_TP026B. pdf $>$
} 


\subsection{Concepção}

Trata-se da primeira fase do ciclo de desenvolvimento de um software - seja ele um programa de computador ou um aplicativo para dispositivos móveis - e tem como objetivo a definição do escopo do projeto. Nesta fase, foi necessário aprofundar os conhecimentos nas áreas de Inclusão e Tecnologia com enfoque em ferramentas para comunicação, com o objetivo de analisar os recursos já existentes e pensar em como desenvolver um recurso com diferencial expressivo.

As ferramentas de CAA têm o objetivo de expandir o repertório de comunicação que está ligado às habilidades de expressão e interpretação. Para tal, são dispostos e confeccionados materiais para auxílio externo, podendo ser cartões de figuras, pranchas de comunicação, do alfabeto, de numerais ou de palavras, vocalizadores ou o próprio computador com software específico pode tornar-se uma ferramenta de voz e comunicação.

Os recursos disponíveis atualmente se dividem em recursos de baixa tecnologia (lowtech) e de alta tecnologia (high-tech). Tal diferença não significa atribuir uma maior ou menor funcionalidade ou eficiência a um ou a outro, mas sim caracterizar apenas a sofisticação dos componentes com os quais esses produtos são construídos e disponibilizados (GALVÃO FILHO; DAMASCENO, 2006).

Os primeiros são aqueles de produção manual, confeccionados com o recorte e a colagem de imagens, palavras, para criar cartões ou pranchas de comunicação ${ }^{2}$, que são uma coleção de símbolos e textos, se adequando à necessidade e à realidade do usuário, podendo ser introduzidas novas figuras e expressões à medida que o seu uso for se naturalizando e tornando intuitivo. Além destes, há também os recursos "de alta tecnologia", que contam com uma variedade maior de opções: utilizam de computadores, tablets, comunicadores por voz gravada e com voz sintetizada.

Esta seção apresenta a etapa de concepção de uma aplicação gratuita para dispositivos móveis que utilizam o sistema operacional Android. Para poder definir o escopo do projeto e o seu diferencial perante os aplicativos já disponíveis, foi necessário, primeiramente, analisar outras aplicações existentes. A pesquisa e download foram feitos na loja de aplicativos PlayStore ${ }^{3}$ utilizando os termos: "aplicativo de comunicação", "CAA" e "Comunicação Alternativa e Aumentativa"; e os critérios de inclusão para definir quais aplicações seriam analisadas foram: (1) disponibilidade para sistema operacional Android e (2) gratuidade. Com isso, foi consultado um total de cinco aplicações ${ }^{4}$, sendo elas: Falaê, Hermes Comunicação Alternativa, PictoTEA, TelepatiX CAA e Matraquinha.

Dessas cinco aplicações consultadas, apenas a Hermes Comunicação Alternativa utiliza somente um teclado em que o usuário digita e em seguida o texto é reproduzido em áudio. Todas as outras quatro aplicações fazem uso de imagens com legendas que são clicáveis e, após o toque, surge a frase ou palavra referente à mesma reproduzida em som. A aplicação aqui

\footnotetext{
${ }^{2}$ Ver páginas 12 a 18 em <http://portal.mec.gov.br/seesp/arquivos/pdf/ajudas_tec.pdf >.

${ }^{3}$ Acessar em <https://play.google.com/>

${ }^{4}$ As aplicações consultadas neste artigo podem ser encontradas nos seguintes links:

Falaê <https://play.google.com/store/apps/details?id=org.falaeapp.falae>,

Hermes comunicação alternativa <https://play.google.com/store/apps/details?id=com.gsampaio.hermes>,

PictoTEA <https://play.google.com/store/apps/details?id=ar.com.velociteam.pictoTEA>,

TelepatiX CAA <https://play.google.com/store/apps/details?id=br.com.geraestec.telepatix> e

Matraquinha <https://play.google.com/store/apps/details?id=com.phonegap.matraquinha>
} 
apresentada utiliza da mesma técnica de imagens com legendas desses quatro aplicativos observados, porém com alguns diferenciais apresentados a seguir, visando à melhor experiência para o usuário.

Todos os cinco aplicativos consultados não possuem possibilidade de inclusão de novas imagens: o usuário só pode utilizar as opções dadas pela aplicação, não podendo assim incluir algo do qual sinta falta. Pensando em atender essa possibilidade de personalização e introdução de novas figuras, foi definido, no escopo do projeto, que o aplicativo Bela Tagarela teria uma categoria chamada "Minhas figurinhas", a fim de aceitar a inclusão de novas figuras feitas a partir de fotos. Os recursos de comunicação de cada pessoa devem ser construídos de forma personalizada, tendo em vista as várias características que atendem as particularidades do usuário.

Muitos dos recursos de alta tecnologia utilizam voz gravada, sendo na maioria das vezes vozes sintetizadas e de um único gênero, ou somente feminina ou somente masculina, sem nenhuma emoção e completamente mecânicas. Dos cinco aplicativos consultados, apenas o Matraquinha não utiliza voz sintetizada, porém, utiliza somente voz feminina. Pensando na importância de gerar naturalidade ao usuário, optou-se por não utilizar vozes sintetizadas e sim fazer a gravação das vozes dos dois gêneros com voluntários.

Ainda se tratando do escopo do projeto, foi definido que o público-alvo para a utilização da aplicação seriam crianças e adolescentes e, para tal, foi planejado um layout simples e bastante ilustrado, para que mesmo uma criança pequena fosse capaz de utilizá-lo guiando-se pelas imagens disponíveis. Vale salientar que a quantidade de opções de imagens dos cinco aplicativos consultados (somente quatro destes trabalham com imagens) é reduzida em relação às duzentas figurinhas criadas, sendo uma média de oitenta e nove imagens por aplicativo consultado. A aplicação com menos imagens é o Hermes Comunicação Alternativa (quarenta e três imagens) e a com mais imagens é o Matraquinha (cento e sessenta e quatro imagens). A decisão de produzir um recurso especificamente para celulares e tablets que não depende da internet para funcionar, partiu do pressuposto de que a CAA deve ser inserida no ambiente natural do indivíduo, sendo esses dispositivos muito utilizados atualmente.

\subsection{Elaboração}

A fase da elaboração é um período crucial é quando acontece a definição do software para que o projeto comece a ser desenvolvido. Inicialmente, foi realizado um levantamento dos requisitos do aplicativo a serem atendidos: o que o software deve fazer, em termos de tarefas e serviços (VAZQUEZ; SIMÕES, 2016). Trata-se de coletar dados e requisições necessárias para que o usuário consiga alcançar seus objetivos (SOMMERVILLE, 2011). Com isso, os requisitos estabelecidos, ou seja, as tarefas que o usuário poderá realizar são: determinar a voz a ser usada na reprodução do som (feminina ou masculina); ouvir as figurinhas separadas por categoria; criar suas próprias figurinhas através de fotos e legendas da sua preferência, para depois poder utilizá-las na categoria "Minhas figurinhas", assim como as figurinhas já disponibilizadas na aplicação; e a possibilidade de enviar feedback sobre o aplicativo em forma de mensagem ou preenchimento do formulário.

Os requisitos não funcionais (ou de qualidade) definem características e limitações do software. Exemplos desses atributos são o tempo de resposta a uma solicitação do usuário (SOMMERVILLE, 2011). Comumente, tais requisitos são de teor mais crítico, pois a averiguação realizada para saber se o aplicativo atende ou não tal requisito é mais complexa, necessitando de testes com possíveis usuários. Sendo assim, foram analisadas partes do 
desenvolvimento do software como performance do aplicativo: trata-se de uma aplicação com muitas imagens, porém sua utilização não prejudica a de outras funções do dispositivo nem a confiabilidade de dados: o aplicativo solicita acesso à câmera para poder criar novas figurinhas e aos alto falantes para poder reproduzir o som, e não necessita de mais nenhum acesso ou dado do usuário para sua utilização (não é necessário nenhum tipo de cadastro para utilizá-lo), manutenibilidade para futuras atualizações e melhorias que possam ser feitas. Todas estas análises foram feitas com o objetivo de tornar o aplicativo funcional e atrativo.

Ao final dessa fase foi criada a documentação da arquitetura do aplicativo (Apêndices A e B), utilizando o Astah Community ${ }^{5}$, software gratuito de modelagem UML (Unified Modeling Language/Linguagem de Modelagem Unificada). Esta documentação é utilizada para guiar o desenvolvimento da aplicação durante a fase seguinte de construção; por meio dela é possível saber o que deve ser desenvolvido a fim de cumprir todas as especificações pensadas para o escopo do projeto durante a sua concepção e os requisitos definidos na etapa de elaboração.

O Diagrama de Casos de Uso (Apêndice A) define o que o usuário pode fazer na aplicação, ou seja, quais módulos ele possui permissão para acessar. Já o Diagrama de Atividades (Apêndice B) define como a aplicação se comporta de acordo com o que é acessado pelo usuário; trata-se de um fluxo que é facilmente alterado de acordo com o comportamento do usuário. Tais diagramas são essenciais para que o período de construção (desenvolvimento) do aplicativo ocorra de maneira eficaz.

\subsection{Construção}

Construção é a etapa que demanda mais tempo, quando o software é desenvolvido até que todos os requisitos anteriormente especificados sejam atendidos. Para isso, foram utilizados os métodos construídos na fase de elaboração. Para o desenvolvimento do aplicativo foi utilizada a IDE (Integrated Development Environment/Ambiente de Desenvolvimento Integrado) Kodular ${ }^{6}$, um ambiente on-line, gratuito e de código fechado, em que todo o desenvolvimento é feito pelo computador e testado instantaneamente em qualquer dispositivo móvel.

Foi utilizada a programação em blocos, com operação intuitiva, ou seja, para criar o layout do aplicativo basta "arrastar" os componentes desejados, como botões, caixa para escrita de textos, imagens, entre outros que se apresentam na forma de elementos visuais, a fim de criar a aparência da tela; a segunda parte, que dará sentido a esses componentes, são os "blocos", elementos encarregados de fazer a comunicação entre todos os comandos, como responder ações e gerar eventos.

Dessa maneira, foi possível visualizar a construção do aplicativo de acordo com cada alteração feita e determinou-se que o design seria simples e intuitivo para que o usuário, independentemente da idade, fosse capaz de manipulá-lo, e, além disso, possuiria legendas e imagens para facilitar o entendimento de cada função criada.

\subsection{Transição}

\footnotetext{
${ }^{5}$ Mais informações em <http://astah.net/editions/community>

${ }^{6}$ Mais informações em <https://www.kodular.io/>
} 
Esta seção diz respeito ao resultado da aplicação desenvolvida, sua utilização e seus principais diferenciais em relação a outras tecnologias para a CAA. Vale ressaltar que a aplicação se encontra disponível para download na loja virtual de aplicativos para dispositivos Android PlayStore ${ }^{7}$. Todas as imagens utilizadas são do Banco de Imagens PngTree ${ }^{8}$, o qual disponibiliza licença paga e ativa para uso comercial de imagens sem ferir direitos autorais dos criadores. O aplicativo, nomeado de Bela Tagarela, também possui Registro no Instituto Nacional de Propriedade Intelectual (INPI) ${ }^{9}$, com a finalidade de garantir autoria e segurança aos desenvolvedores.

Ao iniciar o aplicativo (Figura 1a), a tela que aparecerá possui botões para a página do aplicativo na rede social Instagram ${ }^{10}$ e para a página do Instituto Federal do Triângulo Mineiro (IFTM), pois o desenvolvimento do aplicativo Bela Tagarela e deste artigo são resultados do Trabalho de Conclusão de Curso das autoras na Pós-graduação Lato Sensu em Educação Profissional e Tecnológica Inclusiva desta instituição, além de dois botões ilustrados e a pergunta sobre a voz de sua preferência, com intuito de gerar empatia. Com o objetivo de simplificar o máximo o uso do aplicativo e torná-lo compreensível a todos, optou-se por uma interface simples e colorida sinalizada tanto com texto como com imagens e mensagens de alertas, quando necessário, com poucas opções para não induzir o usuário ao erro.

Figura 1 - Tela Inicial

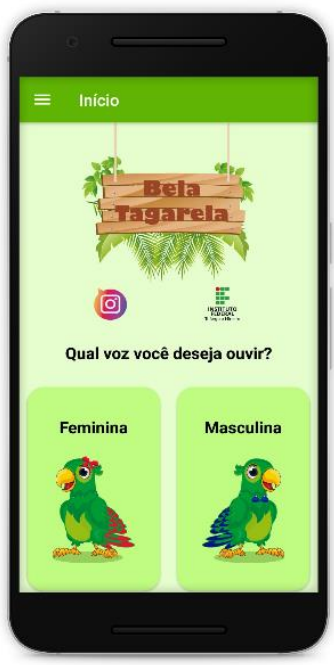

a. Início

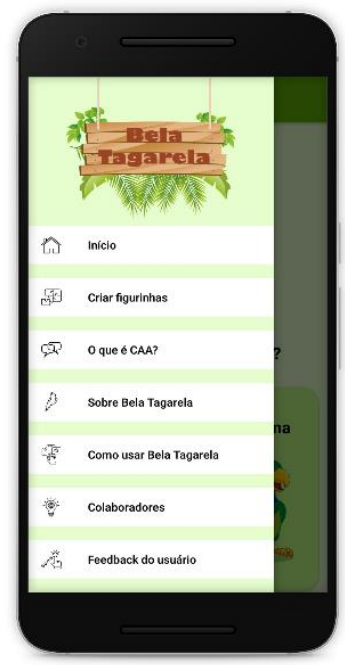

b. Menu lateral

Fonte: Elaborado pelas autoras (2019).

Ainda na tela inicial, ao deslizá-la da esquerda para a direita, aparecerá o menu lateral (Figura 1b), dividido em 7 opções de acesso, sendo elas: (1) Início: (Figura 1a); (2) Criar Figurinhas: (Figura 2); (3) O que é CAA? Tela informativa sobre o que é CAA e a quem se destina o uso do aplicativo; (4) Sobre Bela Tagarela: tela com dados sobre as produções acadêmicas que fundamentaram o desenvolvimento e a consolidação deste aplicativo; (5) Como usar Bela Tagarela: manual sobre como utilizar o aplicativo; (6) Colaboradores: dados dos colaboradores do desenvolvimento, a saber, as autoras desta pesquisa e os voluntários

\footnotetext{
${ }^{7}$ Acessar para baixar o aplicativo Bela Tagarela $<$ https://play.google.com/store/apps/details?id=belatagarela.ta.caa>

8 Acessar em <https://pt.pngtree.com/>

${ }^{9}$ Mais informações sobre registro de software em <http://www.inpi.gov.br/>

${ }^{10}$ Acessar a página do aplicativo no Instagram <https://www.instagram.com/belatagarelacaa/>
} 
responsáveis pelas vozes feminina e masculina e (7) Feedback do usuário: tela de contato para o usuário sugerir melhorias, podendo preencher um formulário ou entrar em contato pelo mensageiro WhatsApp ${ }^{11}$.

Ao clicar na opção do menu lateral de criar figurinhas, uma nova tela se abrirá (Figura 2), onde constarão as informações referentes à quantidade de figurinhas armazenadas no aparelho do usuário, além das opções para criar novas, sendo dividida em duas: (1) Procurar foto no celular/tablet - poderá acessar a galeria de seu aparelho e carregar qualquer imagem que deseje e (2) tirar uma foto com a câmera - a câmera do aparelho será acessada. Após a seleção da imagem ou o registro da nova foto, o usuário deverá nomeá-la e salvá-la, o nome escolhido será o texto falado ao clicar na figurinha posteriormente.

Figura 2 - Criar figurinhas

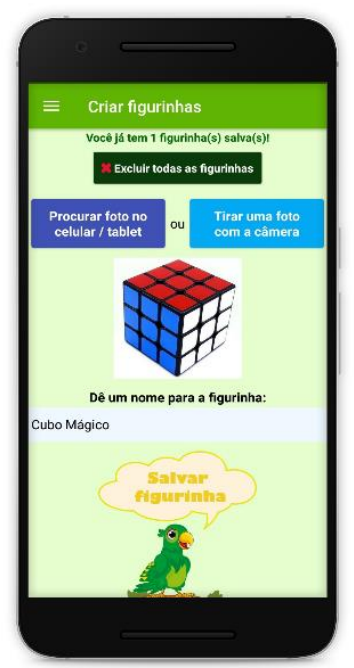

Fonte: Elaborado pelas autoras (2019)

A CAA é usada para demonstrar anseios, indigências, opiniões, afirmações, cumprimentos, sentimentos, desejos, pensamentos, necessidades, pedidos e ideias, entre outros conteúdos de comunicação necessários no cotidiano (CESA; MOTA, 2017). Pensando nisso, após selecionar a voz que deseja ouvir, o usuário terá acesso a dezoito categorias (Figura 3), sendo dezessete categorias com figurinhas pré-determinadas (totalizando duzentas figurinhas) e uma categoria personalizável chamada "Minhas Figurinhas", onde estarão armazenadas todas as figurinhas criadas anteriormente (Figura 2). Ao entrar em uma categoria, basta o usuário clicar sobre a figurinha para poder escutá-la; há na aplicação diversas figurinhas sobre temas variados para tornar a comunicação o mais efetiva possível.

${ }^{11}$ Acessar em <https://www.whatsapp.com/> 
Figura 3 - Categorias
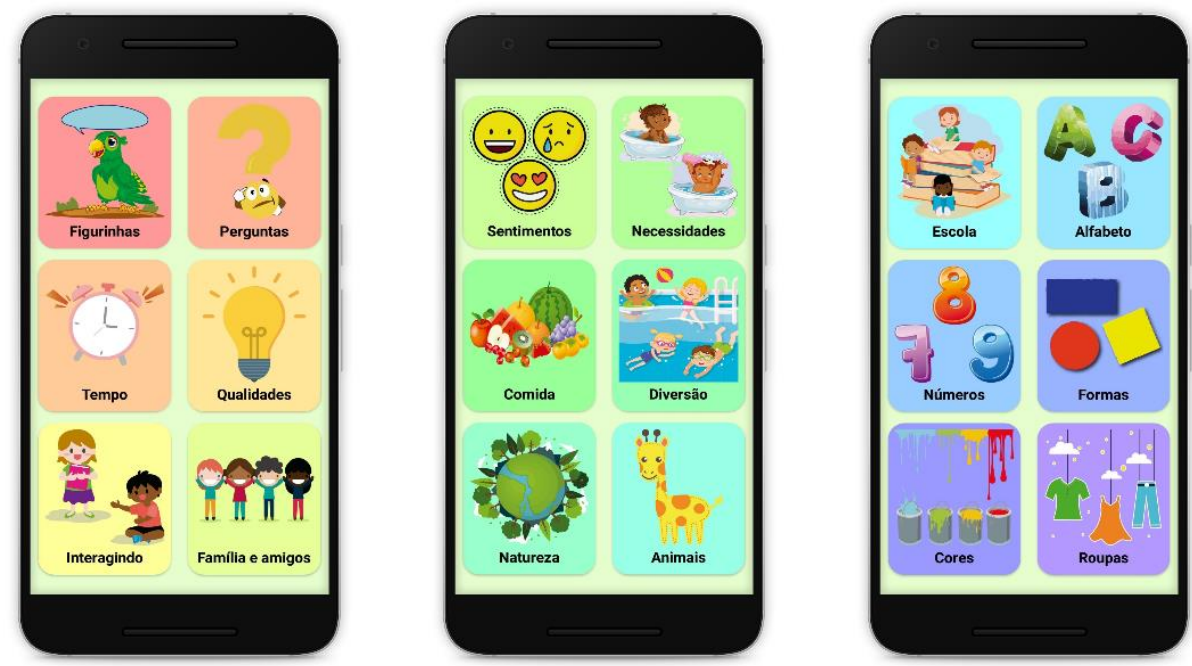

Fonte: Elaborado pelas autoras (2019)

\section{Conclusão}

Este trabalho resultou no desenvolvimento de uma aplicação de CAA para dispositivos móveis, utilizada como alternativa para outras ferramentas de TA de comunicação já existentes, sendo um aplicativo atrativo e de fácil aprendizado, independentemente da idade do usuário. Pensando além da função comunicativa, a CAA auxilia o desenvolvimento das habilidades motoras, cognitivas e afetivas. Conforme afirma (ZAPOROSZENKO; ALENCAR, 2008), ao trabalhar (ou criar) com o sistema de comunicação, o profissional pode e deve dar ênfase nas habilidades motoras tais como: lateralidade, estruturação e organização espacial, tônus, postura, equilíbrio e coordenação dinâmica manual. No que diz respeito às habilidades cognitivas, o desenvolvimento de habilidades referentes à percepção, atenção, memória, raciocínio, conceituação, linguagem e alfabetização também é contemplado utilizando-se CAA.

Em virtude do que foi mencionado, os resultados provenientes do desenvolvimento desta pesquisa são relevantes para a área da Inclusão, sendo capazes de proporcionar uma opção gratuita e portátil para auxiliar no processo de comunicação e com diferenciais como distinção de voz por gênero, inclusão de figurinhas para personalização e maior opção de imagens disponibilizadas em relação às aplicações consultadas. Concomitantemente a esse processo, a aplicação desenvolvida, devido à sua estrutura, contribui para melhorar a autoestima da criança/adolescente possibilitando a participação nas atividades sociais de forma mais facilitada.

Do ponto de vista científico, as principais contribuições deste trabalho são o desenvolvimento de uma aplicação capaz de gerar comunicação através das duzentas figurinhas divididas em dezessete categorias e a possibilidade de inclusão de novas imagens do interesse do usuário; a promoção da utilização e desenvolvimento de tecnologias de baixo custo para a Inclusão e a possibilidade de comunicação utilizando dispositivos móveis que já estão disseminados na sociedade atual.

Espera-se que o desenvolvimento do aplicativo Bela Tagarela e este artigo sejam apenas o começo de uma pesquisa aprofundada sobre a CAA. Para tal, pretende-se, com trabalhos futuros, realizar testes empíricos no aplicativo com pessoas impossibilitadas de falar a fim de validar e aprimorar o uso deste recurso, além de implementar no aplicativo a possibilidade de compartilhamento de figurinhas entre os usuários e também torná-lo acessível para pessoas que, 
além da impossibilidade na fala, também possuam alguma dificuldade motora para manusear celulares ou tablets, como é o caso de pessoas com paralisia ou AVC.

\section{Agradecimentos}

As autoras agradecem aos voluntários, pelo tempo disponibilizado para a colaboração na gravação dos áudios utilizados no aplicativo Bela Tagarela. Pessoas como vocês fazem a diferença.

\section{Referências}

AMERICAN SPEECH-LANGUAGE-HEARING ASSOCIATION. American SpeechLanguage-Hearing Association, 2019 Disponível em: <https://www.asha.org/>. Acesso em: 1 set. 2019.

ARGYLE, M. A interação social: relações interpessoais e comportamento social. Rio de Janeiro: Zahar, 1976.

CESA, Carla Ciceri; MOTA, Helena Bolli. Augmentative and alternative communication: from qualification to speech, language and hearing science clinical practice. Revista CEFAC, v. 19, n. 4, p. 529-538, 2017.

FONTE, Igor Calabria Da. Uma Ferramenta para Personalização de Vocabulário de Prancha de Comunicação Alternativa. 2016. 46 f. Monografia (Bacharelado em Ciências da Computação). Centro de Informática. Universidade Federal de Pernambuco - UFPE, 2016.

GALVÃO FILHO, Teófilo Alves; DAMASCENO, Luciana Lopes. Tecnologias Assistivas para autonomia do aluno com necessidades educacionais especiais. Inclusão: Revista da Educação Especial, p. 25-32, 2006.

KRUCHTEN, P. The Rational Unified Process: An Introduction. 3 ed. ed. Boston: Addison-Wesley, 2004. (The Addison-Wesley object technology series).

LAUAND, G. B. A. Fontes de informação sobre tecnologia assistiva para favorecer à inclusão escolar de alunos com deficiências físicas e múltiplas. 2005. $224 \mathrm{f}$. Tese (Doutorado em Educação Especial). Programa de Pós-graduação em Educação Especial. Universidade de São Carlos, 2005.

SANTAROSA, Lucila et al. Tecnologias Digitais Acessíveis. Porto Alegre: JSM Comunicação Ltda, 2010.

SOMMERVILLE, Ian. Engenharia de Software. 9. ed. São Paulo: [s.n.], 2011.

VAZQUEZ, Carlos Eduardo; SIMÕES, Guilherme Siqueira. Engenharia de Requisitos: Software Orientado ao Negócio. [S.1.]: 2016, 2016.

ZAPOROSZENKO, Ana; ALENCAR, Gizeli Aparecida Ribeiro De. Comunicação Alternativa e Paralisia Cerebral: recursos didáticos e de expressão. Caderno Pedagógico Série: Educação Especial, p. 30, 2008. 
Revista de Educação, Ciência e Tecnologia

\section{Apêndices}

Apêndice A - Diagrama de Casos de Uso

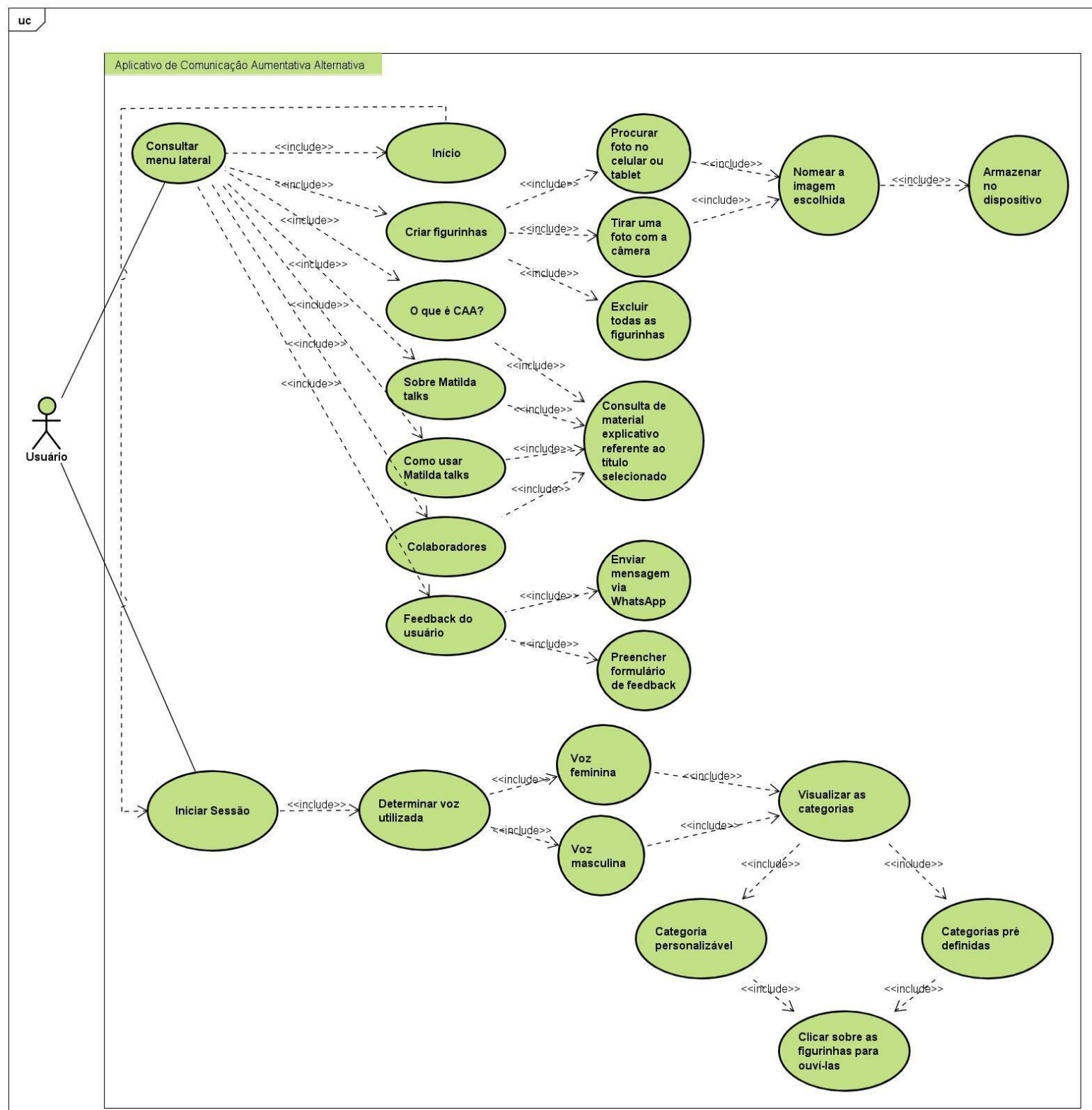

Fonte: Elaborado pelas autoras (2019) 
Apêndice B - Diagrama De Atividades

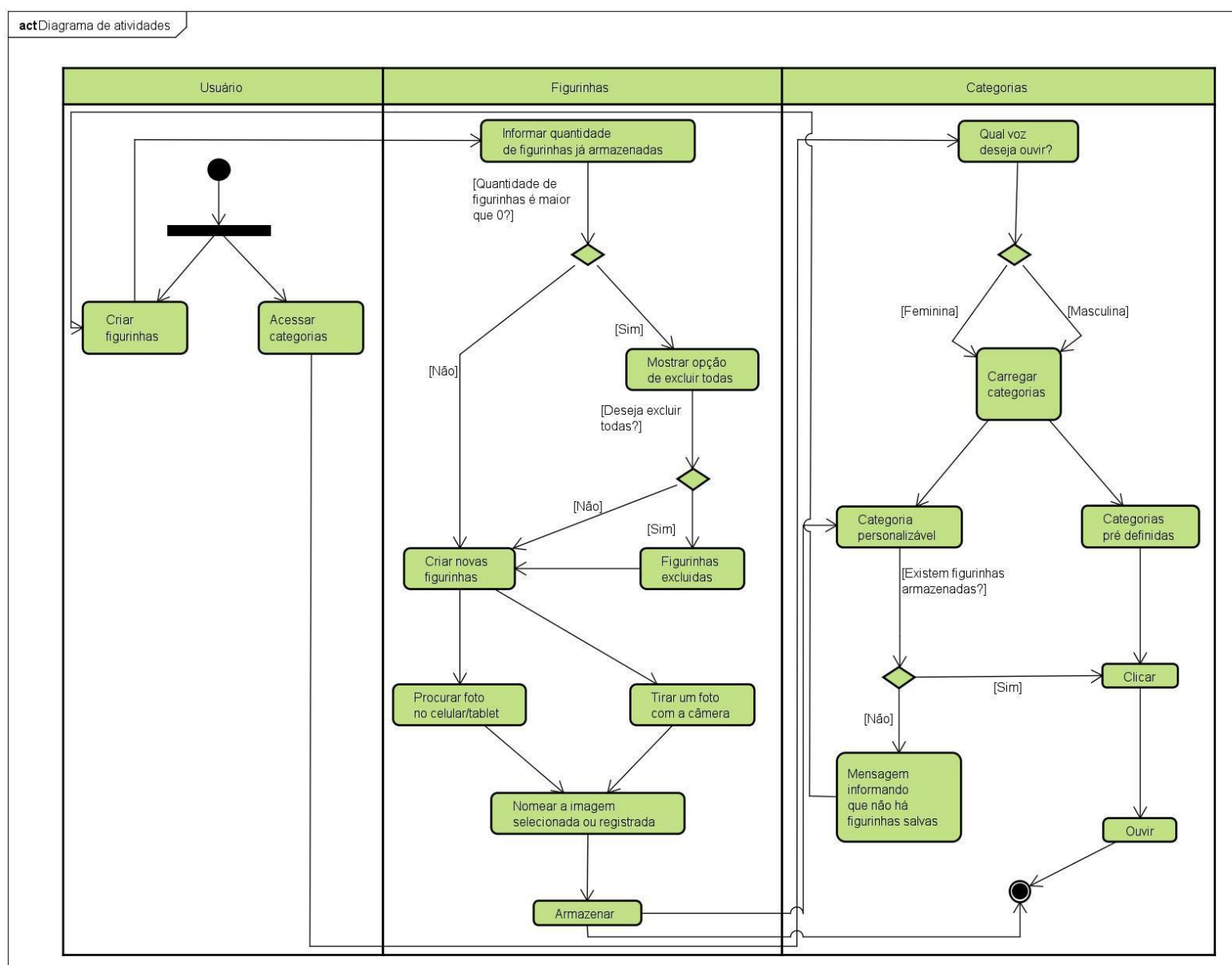

Fonte: Elaborado pelas autoras (2019)

Recebido em março de 2020.

Aprovado em maio de 2020. 